\title{
Comment on "Spain in the Euro: A General Equilibrium Analysis" by Andrés, Hurtado, Ortega and Thomas
}

\author{
Jesús Vázquez
}

Published online: 19 February 2010

(C) The Author(s) 2010. This article is published with open access at Springerlink.com

Is Spain (still) different? This paper addresses this question from a macroeconomic perspective. After reporting differences in rates of output growth and inflation between Spain and the rest of the European Monetary Union (EMU) countries during last decade, the paper suggests three complementary explanations for those differences:

- Different types of shock processes have hit the Spanish economy and the rest of the EMU.

- Different structures leading to different price and wage nominal rigidities and different price and wage indexation mechanisms.

- The common monetary policy may have exacerbated, rather than offsetting, the effects of shocks if they are different in Spain and in the rest of the EMU; and/or if their effects are transmitted differently due to different economic structures.

The paper uses a state-of-the-art, medium-large scale DSGE model known as BEMOD to assess the importance of each of these three explanations. BEMOD considers (i) three geographical areas (Spain, the rest of the EMU and the rest of the world, the latter is assumed to be exogenous), (ii) three production sectors: durable, tradable and non-tradable goods, (iii) nominal price and wage rigidities, (iv) variable capital utilization, and (v) investment adjustment costs. Parameter values are chosen by following a mix of calibration and estimation strategies. Steady-state ratios, preference and technology parameters are calibrated. The remaining parameters are estimated using Bayesian techniques. A secondary goal of the paper is to analyze the performance of BEMOD in several important dimensions: in-sample fit of output growth and inflation, impulse response, variance decomposition and second moment

This commentary refers to the article available at: doi:10.1007/s13209-009-0015-6.

\footnotetext{
J. Vázquez ( $\square)$

Universidad del País Vasco, Bilbao, Spain

e-mail: jesus.vazquez@ehu.es
} 
statistics. By carrying out counter-factual exercises, the paper finds that each of the three explanations plays a role in explaining output growth and inflation differentials between Spain and the rest of the EMU. First, growth and inflation differentials would have been rather different if the Spanish economy had been hit by the same shocks as those estimated for the rest of the EMU. Second, given the historical shocks, removing the differences related to nominal rigidities and indexation mechanisms would have implied a smoother inflation differential. Finally, Spain's membership of the EMU is also having an impact on output growth and inflation differentials.

My comments are organized as follows. I first provide a brief general assessment of the paper's contribution. I then discuss some issues related to the empirical regularities addressed in the paper. Finally, I make a few observations on calibration and estimation issues and on the empirical results.

This paper clearly contributes to our understanding of inflation and growth differentials between Spain and its EMU partners. It uses a well-crafted DSGE model with Keynesian features together with a sound calibration of model parameters. I believe the empirical findings and the conclusions reached in the paper will be taken as benchmarks when both larger sample periods and alternative modelling assumptions are considered in future studies.

The paper motivates its goal simply by showing (Figure 1) inflation and output growth differentials between Spain and the rest of the EMU countries. The question is whether these differentials can be considered as an empirical regularity. Are they large, significant and persistent? I believe there is a lack of discussion on the importance of these differentials. I acknowledge that the short sample period analyzed is an important limitation in identifying any empirical regularity in this context, but more discussion along these lines would be helpful. For instance, the empirical evidence suggests a (weak) positive relationship between output growth and inflation differentials that looks like an aggregate supply schedule expressed in differentials. This feature may suggest that demand shocks (i.e. those that allow us to identify a supply curve) are relatively more important than supply shocks in both Spain and the rest of the EMU members. However, the paper looks at the two empirical regularities of output growth and inflation differentials as being independent outcomes. If the observed cross-covariances at different leads and lags between output growth and inflation differentials were reported, more would be learnt about the potential relationship between these two differentials.

As mentioned above, the paper uses a calibration-estimation approach to choose parameter values. It would be helpful to compare the results obtained from a standard calibration exercise (for instance, using the priors used in the Bayesian estimation procedure implemented) with those obtained from the (posterior) Bayesian estimates. This comparison would allow the contribution of the econometric approach to be assessed with a view to explaining, in this case, output growth and inflation differentials. Moreover, the authors report that the estimated model fits the data in some important dimensions, but they are also honest enough to report that the model fails to reproduce the observed output growth and inflation volatilities or the autocovariance and cross-covariances at different leads and lags. It would be interesting to study in a subsequent paper whether the conclusions reached in this paper still hold when instead of maximizing the likelihood function of the model as is done in this paper, the 
estimation approach follows a generalized method of moments approach where the distance function includes output growth and inflation volatilities as well as the autocovariance and cross-covariances at different leads and lags. Given the main question addressed in this paper I think it is important that the model should be able to do a reasonable job in reproducing second moments of output growth and inflation.

Following Smets and Wouters (2007) the paper obtains the historical time series of alternative shocks to assess the relative importance of those shocks in determining the alternative endogenous variables. I have two main concerns. First, the estimated historical time series of shocks include both identified economic shocks and estimated errors. The paper, however, seems to overemphasize the evidence provided by estimated shocks and ignores the presence of estimated errors. The importance of estimated errors can be studied by assessing how robust the estimated shocks are when considering alternative specifications or alternative parameter values. For instance, would the historical time series (i.e. one step ahead errors) have been much different if the prior means were used instead of the posterior estimates? Second, the method used for calculating the contribution of each particular type of shock to the observed series is not explicitly indicated in the paper.

The paper's main finding is that all three hypotheses suggested for explaining output growth and inflation differentials play a role. It would be interesting for future research to develop a measure in order to assess the relative importance of each of the three hypotheses.

Open Access This article is distributed under the terms of the Creative Commons Attribution Noncommercial License which permits any noncommercial use, distribution, and reproduction in any medium, provided the original author(s) and source are credited.

\section{Reference}

Andrés J, Hurtado S, Ortega E, Thomas C (2010) Spain in the Euro: a general equilibrium analysis. SERIEs 1:67-95

Smets F, Wouters R (2007) Shocks and frictions in U.S. business cycles: a Bayesian DSGE approach. Am Econ Rev 97:586-606 